\title{
DEVELOPMENT OF HYDROGEL CAPSULES FOR USE IN DUAL-PHASE TOPICAL PREPARATIONS CONTAINING DIHYDROXYACETONE AND AMINO ACIDS - PRELIMINARY RESULTS
}

\author{
BIANKA JACYNA', BARBARA MIKOLASZEK', BARTOSZ MACIEJEWSKI', \\ and KRZYSZTOF CAL ${ }^{1 *}$
}

'Department of Pharmaceutical Technology, Medical University of Gdansk, Hallera 107, 80-416 Gdańsk, Poland

\begin{abstract}
The aim of the following work was a preliminary assessment of the possibility of producing a topical preparation containing dihydroxyacetone (DHA) and amino acids, encapsulated separately in polysaccharide capsules. The color reaction between the DHA and amino acids would be possible after mixing of the two components triggered with mechanical disruption of the capsules on application. Such preparation could be used in patients suffering from vitiligo, by masking the symptoms of the disease. Several capsule compositions were evaluated in terms of appearance, dimensions and mechanical parameters. In addition, the reactions of various amino acids with DHA were carried out and obtained colors were analysed. The results indicate that formation of the dual-phase formulation of encapsulated DHA and amino acids is possible. It was also found that certain amino acids vary in color resulting from reaction with DHA. This feature can be potentially utilized to better design the color resulting from application of the prepared composition.
\end{abstract}

Keywords: hydrogel capsules, topical preparations, sodium alginate, gellan gum, amino acids, dihydroxyacetone

The encapsulation process is widely used in both pharmaceutical and cosmetic industries $(1,2)$. The encapsulation ensures separation of the substances from the external environment, however, such substances can be released from the capsules according to several mechanisms, i.e. diffusion, dissolution or mechanical damage of the shell. Various substances can be utilized in formation of capsule shells, including natural polymers, i.e. polysaccharides. The examples of such polymers can be sodium alginate, carrageenan or gellan gum. Sodium alginate is a sodium salt of alginic acid - a biopolymer obtained from brown algae. Its chemical structure comprises homopolymeric blocks of mannuronate and guluronate. Sodium alginate is soluble in water, however, crosslinking reaction of copolymers by ionic bonds between alginate random-coil chains and divalent cations leads to formation of ordered dimeric structure of alginate molecules, resulting in strong gels. Carrageenan is a sulfated linear biopolymer obtained from red seaweed, composed of galactose and anhydrogalactose units. In the following work, a $\beta$-carrageenan was used, due to its potential to react with calcium ions, wchicht results in aggregation of carrageenan helices and formation of gels. Gellan gum is an anionic polysaccharide derived from Sphingomonas elodea bacteria, also forming strong gels in the presence of divalent cations. The reaction between gellan and divalent ions is based on direct interaction of ions on gellan segments with formation of ordered structure, however, it has a complicated nature (3-6). The mechanism that leads to formation of capsule shell can be crosslinking of the polysaccharide chains in the presence of metal ions, where as a result a firm gel-structure is created (7-9). Several polymers under the mentioned conditions can form water-insoluble gels, those exhibit properties desired in the formulation of stable multi-component topical preparations, such as self-tanning cosmetics. The release of encapsulated substances in such composition would be based on mechanical disruption of capsule shell.

Dihydroxyacetone (DHA) is a substance commonly used in cosmetics as a self-tanning agent. DHA reacts with the amino acids located in the stratum corneum and creates brown pigments causing

\footnotetext{
* Corresponding author: e-mail: kcal@wp.pl
} 
coloration of the skin without exposure to sunlight, with a complex series of reactions: starting with deprotonation of the amino group in amino acid, followed by condensation with DHA, dehydration with creation of a Schiff base, than rearrangement to a Heyns product. Subsequent series of reactions, involving additional molecules, eventually lead to the creation of a high molecular weight pigment (10, 11). Based on current literature data, amino acids particularly responsible for triggering a color reaction with dihydroxyacetone are arginine, glycine, lysine, serine, proline, histidine, tryptophan, methionine and ornithine. The reaction of DHA with amino acids can potentially be utilized in masking off areas with changed skin coloration in vitiligo patients, thus improving their quality of life.

Vitiligo is an idiopathic chronic skin disease characterized by patches of the skin losing their pigment. The patches of skin affected become white and usually have sharp edges. The area of the affected skin are often exposed (e.g. hands, legs, face) and more noticeable in people with dark skin. Vitiligo occurs in $1-2 \%$ of the global population, regardless of ethnicity, sex or age. In many cases, the disease finally leads to various psychological disorders because it limits activities of the patients in various spheres of life, i.e. social interactions. There is no known cure for vitiligo, but there are non-invasive methods including cosmetic correction of the diseased skin areas or makeup camouflage $(12,13)$. The correction of the skin tone in diseased skin fragments can be made with cosmetics containing DHA (14). However, to our best knowledge, there is lack of published experimental data on the development of topical preparations with both, amino acids and DHA that could be used in the symptomatic treatment of vitiligo in various skin shades.

Therefore, the aim of the following proof-ofconcept study was to assess possibility of formulation of a topical dosage form comprising two separately encapsulated types of substances, i.e. DHA and amino acids, in the capsules prepared from natural polysaccharides. The reaction between these components in such preparation would be possible after mechanical disruption of the capsules by specially designed dispenser, therefore the capsules should exhibit proper mechanical properties. The second aim of the present study was to compare coloring effect caused by the reaction of DHA with selected amino acids and to compare physical properties of gel capsules obtained with several polymers and intended as a carrier for skin coloring agent. The results will allow optimizing of key parameters of water-insoluble gel capsules intended for applica- tion with dedicated dispensers, assuring mechanical damage to the capsules and release the DHA included in the fill.

\section{EXPERIMENTAL}

\section{Materials}

Gellan gum (Phytagel) and amino acids (arginine, glycine, lysine, serine, proline, histidine, tryptophan, methionine and ornithine) were purchased from Sigma-Aldrich (Darmstadt, Germany). DHA was provided by Merck (Darmstadt, Germany). Sodium alginate (Protanal LF 10/60FT) and carrageenan (Gelcarin GP 379) were obtained from FMC Biopolymer (Philadelphia, USA). Calcium chloride was purchased from Ubichem (Redditch, UK).

\section{Preparation of amino acids and DHA solutions}

The solutions of amino acids were prepared by dissolving the amino acid powders in purified water under magnetic stirring, to obtain a final concentrations of $0.5-5.0 \%$. The DHA solutions were prepared by dissolving the DHA in purified water to obtain final concentration of $2-10 \%$. Solutions were placed in glass vials with a tight screw cap and used for further testing within a few hours.

\section{Saturation of cellulose membranes}

Cellulose discs with a diameter of approx. 5.5 $\mathrm{cm}$ were saturated with aqueous solutions of the following amino acids: arginine (Arg), glycine (Gly), lysine (Lys), serine (Ser), proline (Pro), histidine (His), tryptophan (Try), methionine (Met) and ornithine (Orn). The tested concentration range was $0.5-5.0 \%$. After saturation, the cellulose discs were dried and saturated with varying concentrations of DHA aqueous solutions in a concentration range of $2-10 \%$.

The DHA-saturated cellulose discs were placed on glass plates and stored in a climate chamber $\left(25^{\circ} \mathrm{C}, 60 \% \mathrm{RH}\right)$ for 3 days. After each $24 \mathrm{~h}$ change in color was observed.

\section{Color analysis}

Dry cellulose films were scanned with an office scanner (HP Scanjet G3110, HewlettPackard, USA), using white sheet as a background. After scanning, the images were divided into 9 subsections equal in size. The central subsection, as the one furthest from the edges of the disc, was subjected to color analysis. This protocol allowed avoiding analysis of sections containing artificially more intensive coloration, due to higher deposition of 
investigated substances near the edges of cellulose discs. The images were analyzed using ImageJ 1.51 software (National Institutes of Health, USA), 3 basic color layers were extracted according to RGB (Red Green Blue) color classification model. The intensity of each color was analyzed in terms of uniformity of shade, distribution of intensity and what is the proportion of this color in formation of the final complex dye. The results of the analysis were

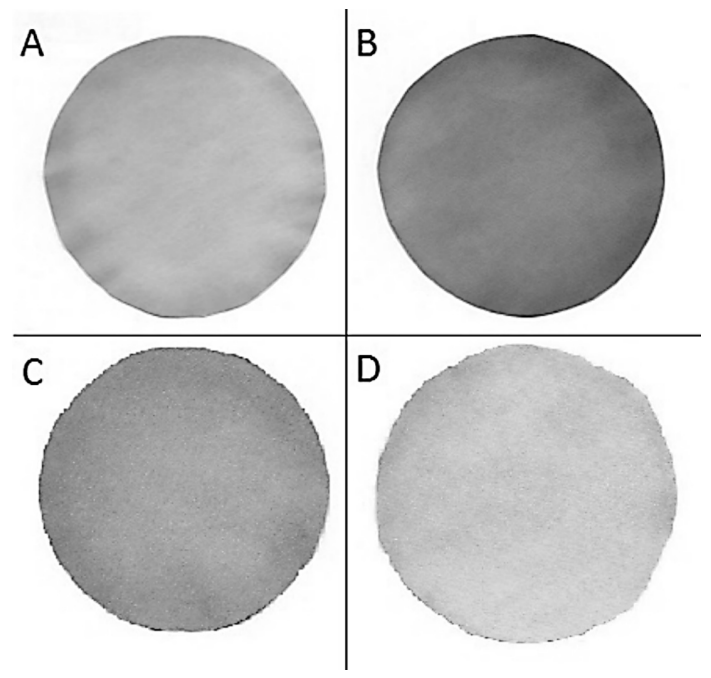

Figure 1. Scanned images of membranes impregnated with 5\% Arg and 10\% DHA (A), 5\% Gly and 10\% DHA (B) (both cured for $24 \mathrm{~h}$ at $\left.25^{\circ} \mathrm{C}, 60 \% \mathrm{RH}\right) ; 5 \% \mathrm{His}$ and $10 \%$ DHA (C) and $5 \%$ Orn and $10 \%$ DHA (D) (both cured for $72 \mathrm{~h}$ at $25^{\circ} \mathrm{C}, 60 \% \mathrm{RH}$ ) expressed with histogram and numerical parameters. Comparison of intensity and uniformity of each color component between formulations was performed.

\section{Preparation of hydrogel capsules}

Three different polysaccharides were used to prepare gel capsules: sodium alginate (Protanal LF 10/60FT), carrageenan (Gelcarin GP 379), and gellan gum (Phytagel).

Polymer solutions were obtained by dissolving the polysaccharide in warm purified water under constant stirring. The solutions were added dropwise into a calcium chloride solutions that varied in the concentration of calcium ions, under constant stirring. After a few seconds obtained capsules were separated from the stirred solution by filtration and rinsed with purified water to wash out the excess of crosslinking agent. The capsules were subjected to further analyses instantly after manufacturing.

\section{Size and shape}

The size and shape of gel capsules were evaluated with OptaView X2000 stereoscopic microscope and OptaView 7 software (Opta-Tech, Warsaw, Poland). Besides dimensions, the smoothness of the capsule surface was assessed.

\section{Mechanical properties}

Mechanical resistance of the capsules was evaluated as resistance to stress, by measuring the
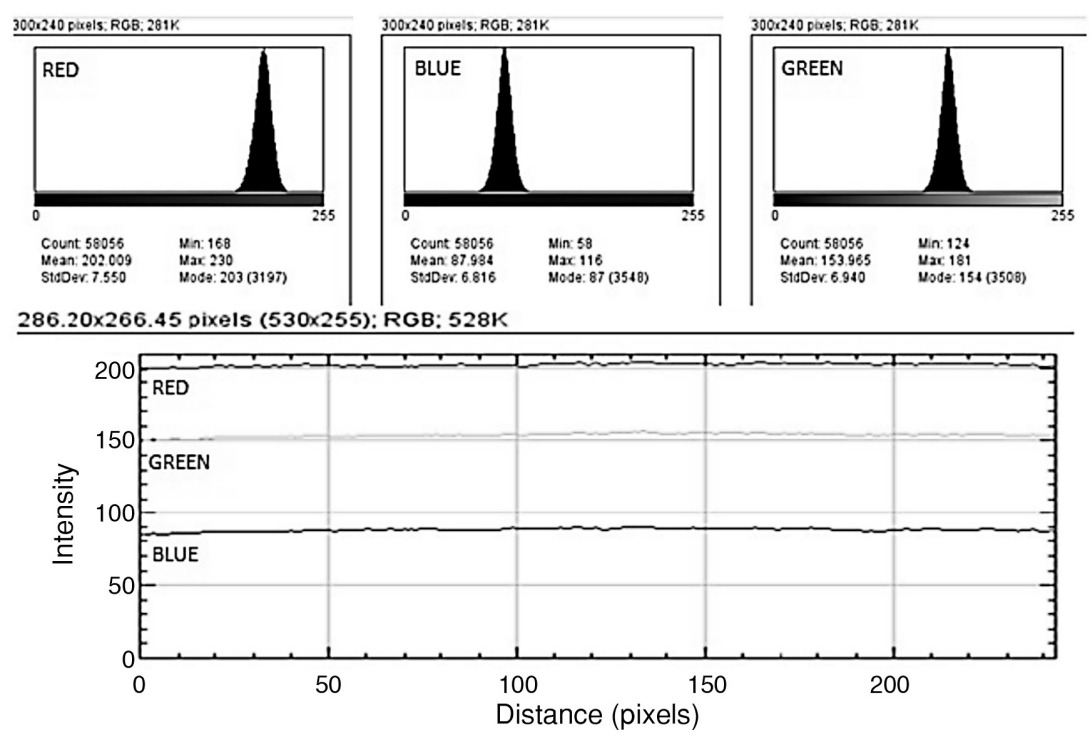

Figure 2. Results of the RGB color analysis and intensity levels of the RGB components of disc impregnated with 5\% of arginine and $10 \%$ of DHA solutions 
force required to apply $80 \%$ strain to the capsule in a compression test. Each formulation was tested using TA.XT Plus texture analyzer (Stable Micro Systems, Godalming, UK) equipped with $\varnothing 6 \mathrm{~mm}$ cylindrical stainless steel probe. The results were calculated using Exponent software (Stable Micro Systems, Godalming, UK).

\section{RESULTS AND DISCUSSION}

\section{Color analysis}

Although the cellulose discs are a very simple model with little to no relation to the structure of human skin tissue, it allowed for preliminary assessment of the concentration ranges and feasibility of
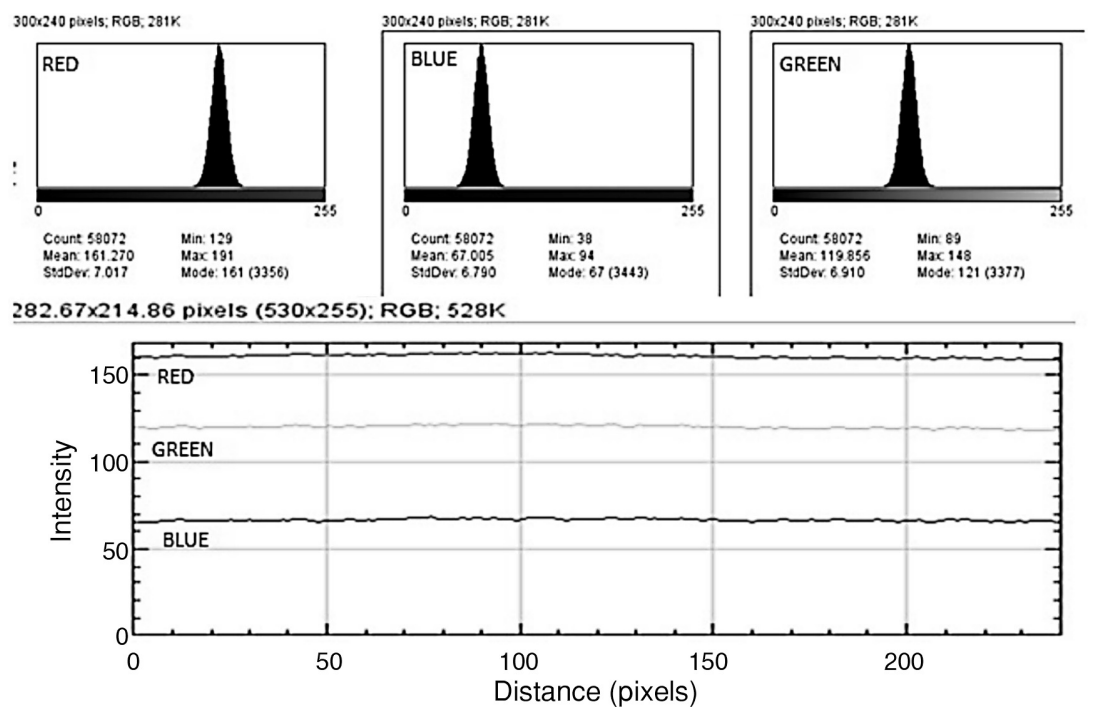

Figure 3. Results of the RGB color analysis and intensity levels of the RGB components of disc impregnated with 5\% of glycine and $10 \%$ of DHA solutions
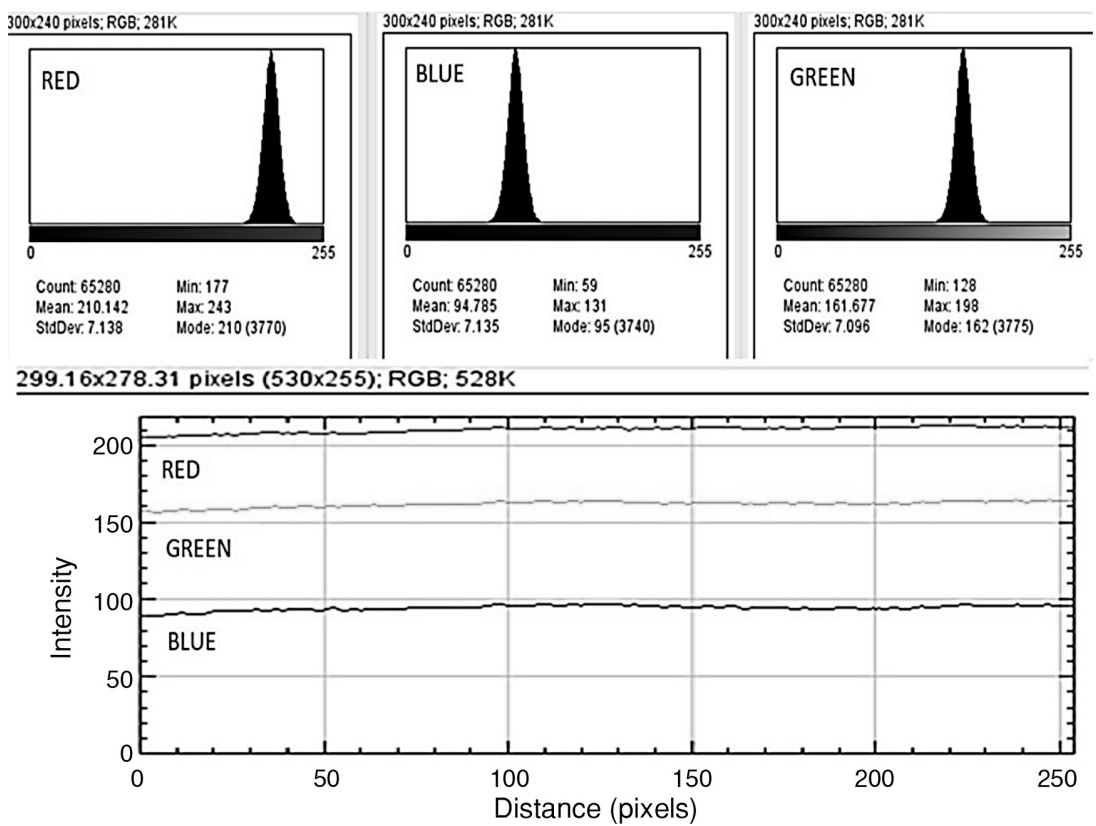

Figure 4. Results of the RGB color analysis and intensity levels of the RGB components of disc impregnated with 5\% of histidine and $10 \%$ of DHA solutions 

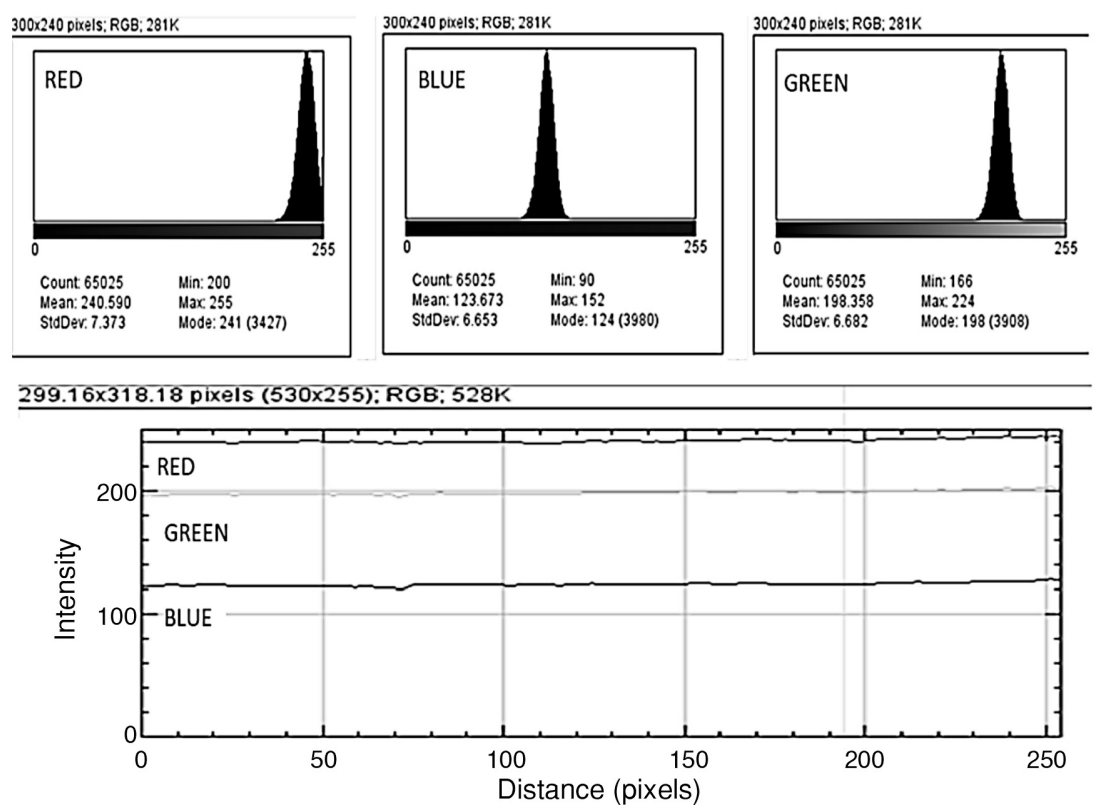

Figure 5. Results of the RGB color analysis and intensity levels of the RGB components of disc impregnated with 5\% of ornithine and $10 \%$ of DHA solutions

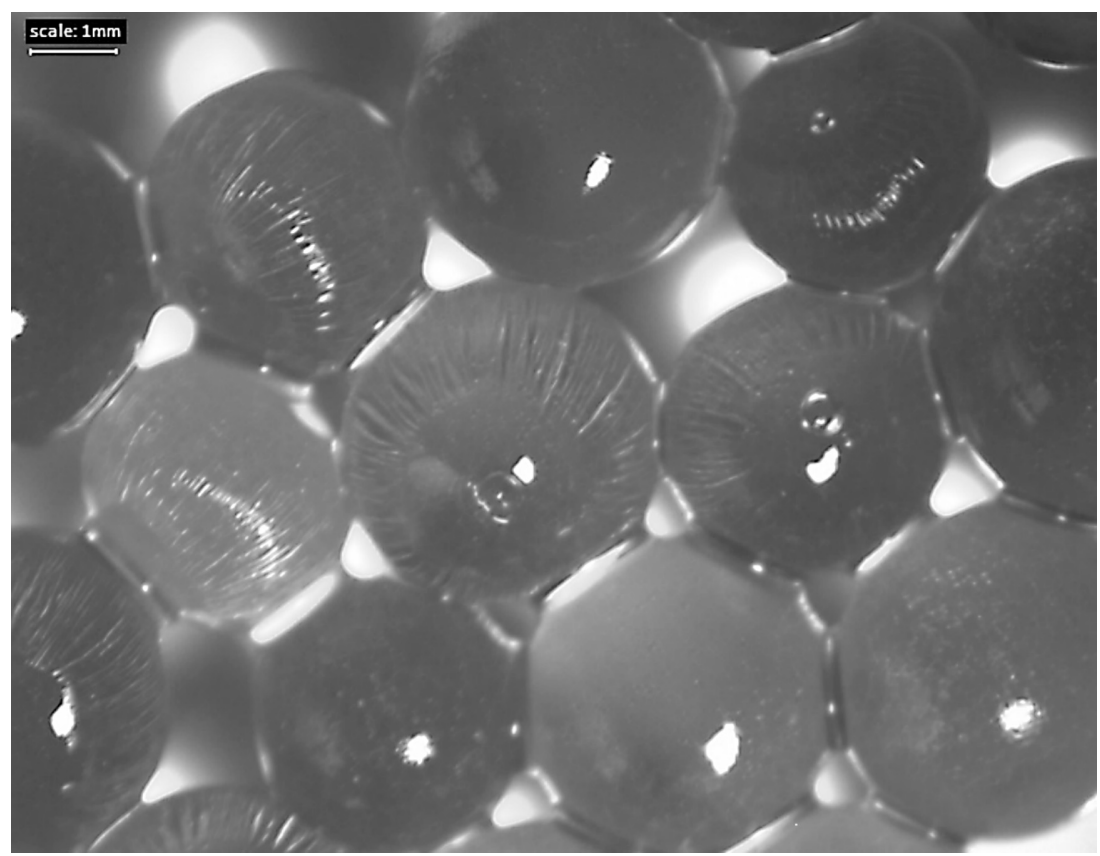

Figure 6. Microscopic image of capsules prepared with $2 \%$ sodium alginate and $1 \% \mathrm{CaCl}_{2}(\mathrm{scale} 1 \mathrm{~mm})$

certain amino acids for further development of the topical preparation. It was found that the shortest time needed to obtain coloration $\left(24 \mathrm{~h}\right.$ at $25^{\circ} \mathrm{C} / 60 \%$ $\mathrm{RH})$ was provided by the discs impregnated with arginine or glycine solutions, when $5 \%$ amino acid and $10 \%$ DHA were used (Fig. 1). Significantly longer curing time was required to obtain coloration of the cellulose discs with $5 \%$ solutions of histidine or ornithine, and $10 \%$ DHA ( $72 \mathrm{~h}$ at $\left.25^{\circ} \mathrm{C} / 60 \% \mathrm{RH}\right)$. Good uniformity of the color in these membranes is evidenced by the fact that no significant fluctuations in the intensity level of the three RGB components were noticed in the entire examined area of each membrane (Figs. 2 and 3). Darker color was 
obtained by reaction of DHA with glycine solution; moreover, both compositions resulted in color with red as the most pronounced component. Similar results were obtained with disk saturated with $5 \%$ histidine solution (Fig. 4). In this case, the intensity level of all three RGB components resulted in a darker tone, quite alike to disc saturated with glycine solution. Comparison between discs saturated with glycine and histidine shows that discs saturated with arginine solution, although having a lighter color, shown all the basic colors had higher intensity. This suggests that the dark tone of obtained colors is derived more likely from the proportion of each RGB component than from high intensity of one or more components. This appears to be supported by the results of the color analysis in the case of cellu-

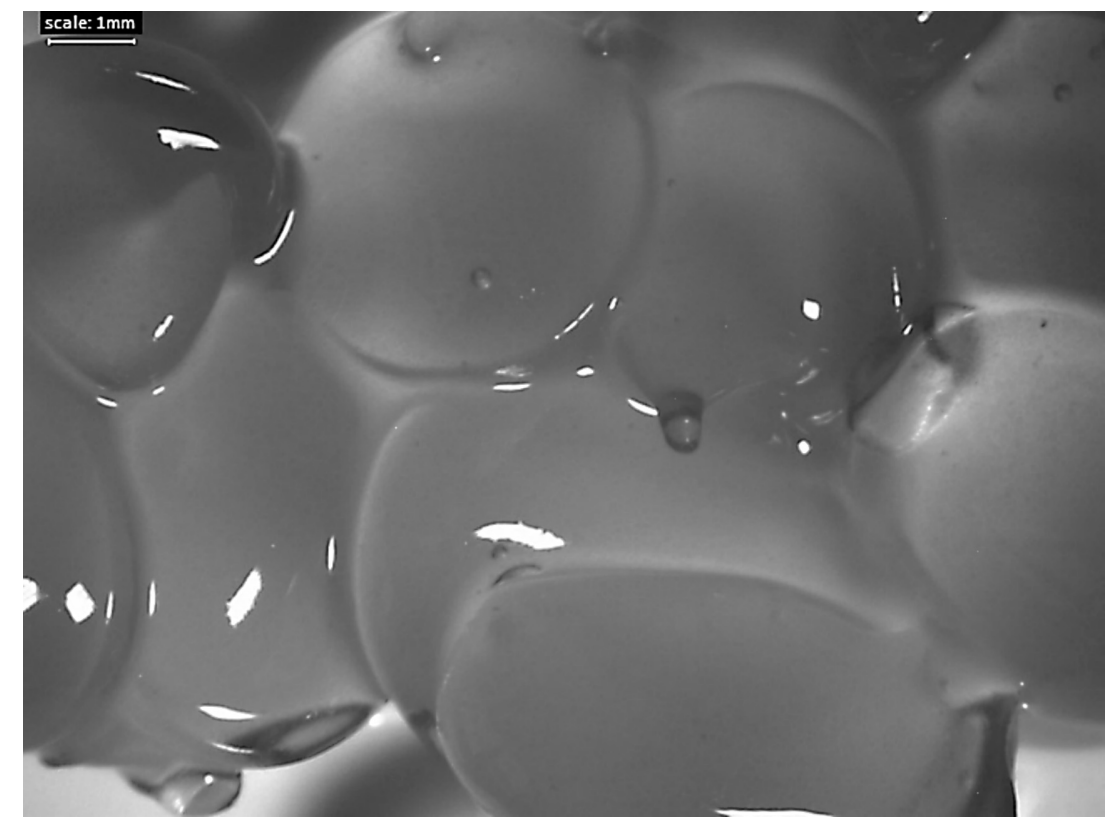

Figure 7. Microscopic image of capsules prepared with $4 \%$ carrageenan and $3 \% \mathrm{CaCl}_{2}$ (scale $1 \mathrm{~mm}$ )

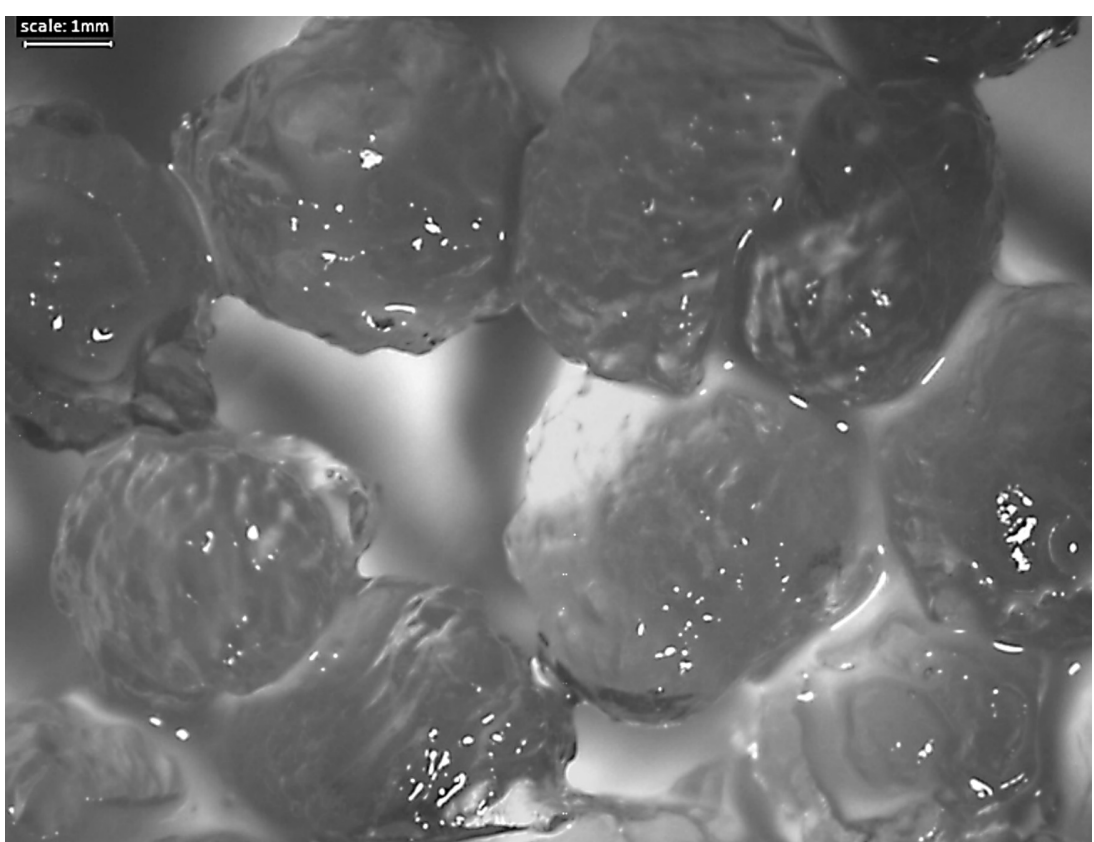

Figure 8. Microscopic image of capsules prepared with $2 \%$ gellan gum and $6 \% \mathrm{CaCl}_{2}$ (scale $1 \mathrm{~mm}$ ) 


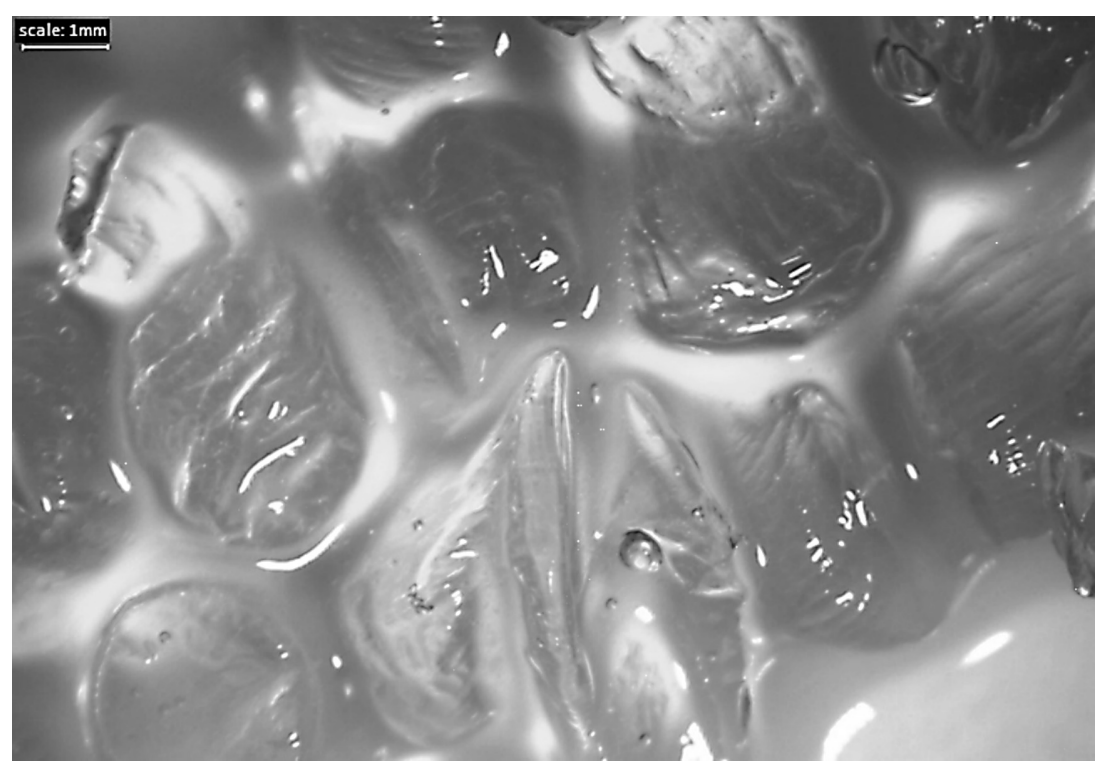

Figure 9. Microscopic image of capsules prepared with $1 \%$ gellan gum and $4 \% \mathrm{CaCl}_{2}$ (scale $1 \mathrm{~mm}$ )

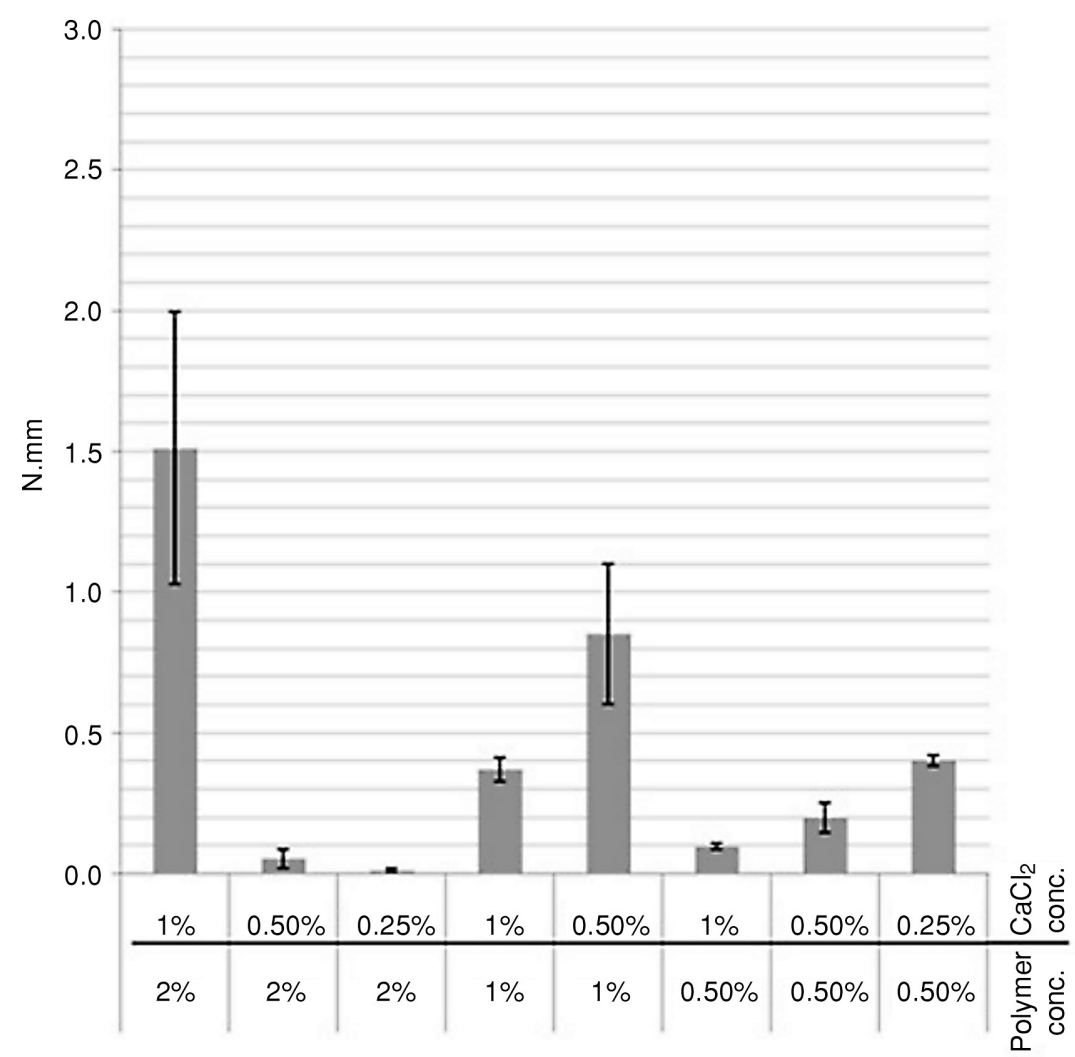

Figure 10. Mechanical resistance of hydrogel capsules made with sodium alginate

lose disc saturated with $5 \%$ ornithine solution, where, despite the highest intensities of RGB components of all discussed samples, the lightest tone of coloration was obtained (Fig. 5). It seems that high- er levels of the red component lead to obtaining a lighter tone, especially combined a with high level the green component. On the other hand, a relatively low level of the blue component appears to be 


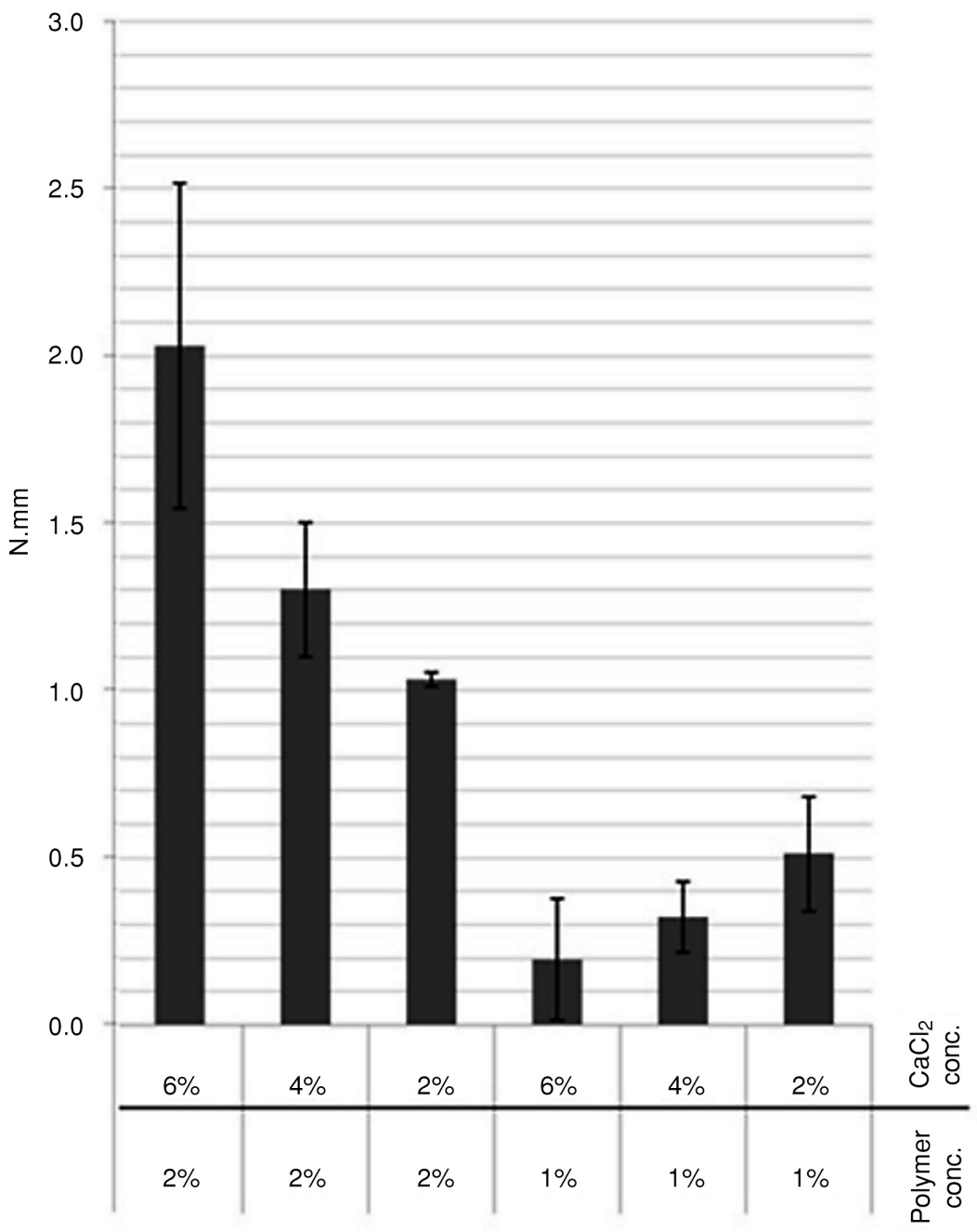

Figure 11. Mechanical resistance of hydrogel capsules made with gellan gum

related to obtaining a darker tone. In both darker samples, i.e. saturated with glycine (Fig. 3) and with histidine (Fig. 4) the intensity of blue component appears to be noticeably lower relative to the other two basic colors. Another issue can be lower uniformity of obtained color in the case of darker tones, especially the disc colored with histidine solution, what can be considered a flaw, but also opens an opportunity of mimicking the naturally occurring differences and imperfections in skin tone. This, however, needs more thorough investigation in order to correlate the obtained color with parameters of color components, derived from the amino acid used in the reaction. Moreover, the cellulose discs are not a very complex model for assessment of potential influence of discussed preparation on the human skin. Therefore, a similar investigation needs to be proceed with the use of human skin tissue (15). Anyhow, based on the obtained results, it can be expected that the color and tone that results from the preparation can be designed specifically for the particular patient by matching the shade of skin coloration to the natural tone of healthy skin in vitiligo patients $(16,17)$.

\section{Capsules}

Out of 27 proposed polymer solutions, 20 allowed the formation of hydrogel capsules in the studied range of polymer concentrations and with calcium chloride as a crosslinking agent. The 
Table 1. Hardness and mechanical resistance of various formulations of capsules.

\begin{tabular}{|c|c|c|c|c|}
\hline $\begin{array}{c}\text { Capsule shell } \\
\text { material }\end{array}$ & $\begin{array}{c}\text { Polymer concentration } \\
{[\%]}\end{array}$ & $\begin{array}{c}\text { Crosslinker } \\
{[\%]}\end{array}$ & $\begin{array}{c}\text { Hardness } \\
{[\mathrm{N}]}\end{array}$ & $\begin{array}{c}\text { Mechanical resistance } \\
{[\text { N.mm] }}\end{array}$ \\
\hline \multirow{4}{*}{ Sodium alginate } & 0.5 & 0.25 & 0.77 & 0.40 \\
\cline { 2 - 5 } & 0.5 & 0.5 & 0.43 & 0.20 \\
\cline { 2 - 5 } & 0.5 & 1.0 & 0.32 & 0.10 \\
\cline { 2 - 5 } & 1.0 & 0.5 & 0.93 & 0.85 \\
\cline { 2 - 5 } & 1.0 & 1.0 & 0.56 & 0.37 \\
\cline { 2 - 5 } & 2.0 & 0.25 & 0.07 & 0.01 \\
\cline { 2 - 5 } & 2.0 & 0.5 & 0.16 & 0.06 \\
\hline \multirow{5}{*}{ Gellan gum } & 2.0 & 1.0 & 1.24 & 1.51 \\
\cline { 2 - 5 } & 1.0 & 2.0 & 0.42 & 0.51 \\
\cline { 2 - 5 } & 1.0 & 4.0 & 0.47 & 0.32 \\
\cline { 2 - 5 } & 1.0 & 6.0 & 0.33 & 0.20 \\
\cline { 2 - 5 } & 2.0 & 4.0 & 0.61 & 1.03 \\
\cline { 2 - 5 } & 2.0 & 6.0 & 0.71 & 1.30 \\
\hline
\end{tabular}

obtained alginate capsules had a diameter between 2.5 and $5.5 \mathrm{~mm}$. It was found that capsules that were closest to the perfectly spherical shape were obtained with sodium alginate $(2 \%)$ and $0.25-1 \%$ of calcium chloride solution (Fig. 6).

On the other hand, the capsules made with carrageenan required $4 \%$ polymer solution and $3 \%$ crosslinker for the best appearance (smooth surface and round to ellipsoid shape) and they reached an average diameter of ca. 4-5 mm (Fig. 7).

Gellan gum capsules reached sizes between 3 and $5 \mathrm{~mm}$ (prepared with $2 \%$ polymer solution), however, were characterized by rough surface and slightly irregular shapes (Fig. 8) over a wide range of investigated concentrations. However, the capsule compositions differed significantly considering mechanical parameters (discussed further in the article). An example of a composition with little application possibilities can be the formulation prepared with $1 \%$ gellan gum and $4 \% \mathrm{CaCl}_{2}$, shown in Figure 9. This composition, despite being essentially similar in terms of appearance to the composition shown in Figure 8, displayed poor mechanical characteristics (18).

\section{Mechanical resistance}

Prior to the assessment of mechanical resistance of capsules, a strain range sweep (60-85\%) was performed in order to select strain value, at which the highest repeatability of force measurements can be achieved. The best repeatability (lowest standard devi- ation) was determined at $80 \%$ strain in case of all tested capsule formulations. The mechanical resistance was calculated as an area under the curve on the graph depicting changes in measured force in a function of capsule deformation. The mechanical resistance can be defined as a derivative of capsule hardness, but also containing data about the pliability of obtained capsule shells and how it reacts to the applied compression. Therefore, these two parameters (hardness and mechanical resistance) are not depicting the same feature of the investigated capsules. The results of the performed analyses are shown in Table 1 .

The results are presented for 14 formulations of obtained gel capsules, comprising alginate and gellan gum, varying in polymer and crosslinker concentrations (Figure 10 - for sodium alginate, Figure 11 - for gellan gum). The highest mechanical resistance was noted for capsules prepared with $2 \%$ gellan gum in a polymer solution and $6 \%$ calcium chloride in crosslinking solution. For alginate capsules, the highest mechanical resistance was obtained with $2 \%$ polymer and $1 \%$ of crosslinking agent. The capsules prepared from this composition also were characterized by the best visual appearance of all the investigated samples (shown earlier in Fig. 6). This composition also showed the highest hardness values that have been measured during the test. Anyhow, said composition shown lower mechanical resistance value than it was determined for certain gellan-based composition. Moreover, the gellan capsules showed two times higher average mechan- 
ical resistance than alginate capsules, with a noticeably higher ratio of mechanical resistance and hardness. Therefore, gellan capsules, although being visually defective, represented better mechanical characteristics than alginate capsules overall. The summary of mechanical resistance values for alginate and gellan capsules is graphically shown in Figures 10 and 11.

The carrageenan capsules proved to be rather soft and gentle, and their resistance to mechanical stress was below the detection limit of the used equipment. Therefore, carrageenan capsules are considered not suitable for the purpose of the following work.

\section{CONCLUSIONS}

The differences between coloring effect caused by reaction of dihydroxyacetone with amino acids can be evaluated using the described method. The fastest coloring effect was observed in the case of membranes impregnated with arginine and glycine solutions (approx. $24 \mathrm{~h}$ ). The best uniformity of the color of cellulose discs was noticed for arginine and ornithine. Optimum concentration of amino acid solutions was $5 \%$, whereas the concentration of DHA that allowed the best coloring effect was $10 \%$. Furthermore, the results indicate the possibility of obtaining a variety of tones and colors to better match the natural skin tone of particular patients, with the use of various amino acids.

The mechanical properties of gel capsules made with polysaccharides depended on polymer concentration and percentage of calcium chloride in crosslinking solution. The satisfactory mechanical resistance was demonstrated by the capsules made of sodium alginate and gellan gum. Capsules with the most desirable features were obtained using a $2 \%$ concentration of, both sodium alginate and gellan gum. The optimum concentration ratio of crosslinker $\left(\mathrm{CaCl}_{2}\right)$ to polymer for alginate and gellan was $1: 2$ (2\% alginate and $1 \%$ crosslinker) and 3 : 1 (2\% gellan and 6\% crosslinker) respectively. Gellan gum allowed obtaining the mechanically resistant capsules over a wider range of concentrations than sodium alginate.

Although, due to the fact that the following study did not comprise encapsulating of mentioned active substances, a follow-up study needs to be performed.
The presented preliminary results indicate the possibility of developing the multiphase topical preparation containing amino acids and DHA, which could be used in the treatment of vitiligo.

\section{REFERENCES}

1. Østberg T., Graffner Ch.: Acta Pharm. Nord. 4, 201 (1992).

2. Casanowa F., Santos L.: J. Microencapsul. 33, 1 (2016).

3. Rowe R.C., Sheskey P.J., Quinn M.E.: Handbook of Pharmaceutical Excipients, Sixth Edition, pp. 122-126, pp. 622-624, Pharmaceutical Press and American Pharmacists Association, Washington 2009.

4. Chan L.W., Jin Y., Heng P.W.S.: Int. J. Pharm. 242, 255 (2002).

5. Michel A-S., Mestdagh M.M., Axelos M.A.V.: Int. J. Biol. Macromol. 21, 195 (1997).

6. Miyoshi E.: Osaka University Knowledge Archive 35, 23 (2009).

7. Mofidi N. Aghai-Moghadam M., Sarbolouki M.N.: Process Biochem. 35, 885 (2000).

8. Takka S., Acartürk F.: Pharmazie 54, 137 (1999).

9. Osmałek T., Froelich A., Tasarek S.: Int. J. Pharm. 466, 328 (2014).

10. Fesq H., Brockow K., Strom K. et al.: Dermatology 203, 241 (2001).

11. Nguyen B.-C., Kochevar I.E.: Br. J. Dermatol. 149, 332 (2003).

12. Popko M., Kacalak-Rzepka A., Bielecka-Grzela S. et al.: Ann. Acad. Med. Stetin. 57, 23 (2011).

13. Ezzedine K., Eleftheriadou V., Hons M.W., N. van Geel: Lancet 386, 74 (2015).

14. Rajatanavin N., Suwanachote S., Kulkollakarn S.: Int. J. Dermatol. 47, 402 (2008).

15. Phung S.L., Bouzerdoum A., Chai D.: IEEE Trans. Pattern Anal. Mach. Intell. 27, 148 (2005).

16. Alaluf S., Atkins D., Barrett K. et al.: Pigment Cell Melanoma Res. 15, 112 (2002).

17. Alikhan A., Felsten L.M., Daly M., PetronicRosic V.: J. Am. Acad. Dermatol. 65, 473 (2011).

18. Coutinho D.F., Sant S.V., Shin H. et al.: Biomaterials 31, 7494 (2010).

Received: 7.01.2019 